\title{
Effects of behavioural activation on substance use and depression: a systematic review
}

\author{
Carmela Martínez-Vispo ${ }^{1 *}$ (D) Úrsula Martínez ${ }^{2}$, Ana López-Durán ${ }^{1}$, Elena Fernández del Río ${ }^{3}$ and Elisardo Becoña ${ }^{1}$
}

\begin{abstract}
Introduction: Substance use and depression co-occurrence is a frequent phenomenon and an important public health concern. Given the clinical implications and the high prevalence of both disorders, effective interventions are needed.

Methods: The aim of this study is to review Behavioural Activation (BA) intervention effects to improve substance use behaviour and depression. A systematic review was conducted using MEDLINE, EMBASE, and PsycINFO. The Effective Public Health Practice Project Quality Assessment Tool (EPHPP) was used to assess the methodological quality of included studies. Two authors independently screened titles and abstracts, reviewed selected studies, and extracted data.

Results: Of the 7286 studies identified, eight met inclusion criteria. Designs of the studies included six randomized controlled trials (RCTs), and two pre-post design studies. One trial received weak methodological quality, six moderate, and one strong. Three studies addressed smoking behaviour; two targeted opiate dependence; two focused on alcohol/ drug dependence; and, one on crystal methamphetamine abuse. Results showed that BA had a positive effect on substance use outcomes in seven of the eight reviewed studies, and improved depression over time in six studies.

Conclusions: Although studies conducted so far are limited by their heterogeneity and sample sizes, results are promising. There is a need of well controlled and powered studies to establish and to confirm the effectiveness of BA for the treatment of substance use and depression. Future studies should include stronger methodological designs, larger sample sizes, and long-term follow-ups.
\end{abstract}

Trial registration: PROSPERO registration number: CRD42016039412.

Keywords: Behaviour therapy, Behavioural activation, Substance use disorder, Depression, Systematic review

\section{Introduction}

Substance use disorders (SUDs) and mental health disorders are significant contributors to the global burden of disease, and their impact is increasing over the years both in high-income and low-to-middle-income countries [1]. In fact, prevalence of SUDs reaches the $8.7 \%$ of U.S. adults, and among the most prevalent mental disorders stand out depression, with about $6.7 \%$ of U.S. adults having a major depressive episode during the past year [2].

In the general population, co-occurrence of SUDs (including alcohol, tobacco, cannabis, cocaine, and other

\footnotetext{
*Correspondence: carmela.martinez@usc.es

${ }^{1}$ Smoking Cessation and Addictive Disorders Unit, Department of Clinical

Psychology and Psychobiology, Faculty of Psychology, University of Santiago de Compostela, Santiago de Compostela, Spain

Full list of author information is available at the end of the article
}

illicit drugs) and depression is a common and well documented phenomenon [2-6]. In this sense, a meta-analysis of epidemiological studies of the comorbidity of SUDs and mood and anxiety disorders [7], found a strong association between major depression and several SUDs such as alcohol use disorders (pooled Odd Ratio [OR] = 2.42 ) and illicit drug use disorder (pooled $\mathrm{OR}=3.80$ ). Similarly, research has shown that people with SUDs is more likely to have a major depressive disorder, compare to those without SUDs, even after controlling for sociodemographic characteristics and additional psychiatric comorbidity (adjusted $\mathrm{OR}=1.2$ and 1.3, respectively) [8]. Moreover, depression has also been found consistently higher in smokers compared to never smokers $(\mathrm{OR}=$ $1.50)$ and former smokers $(\mathrm{OR}=1.76)[9]$.

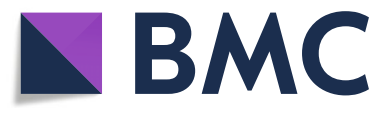

(c) The Author(s). 2018 Open Access This article is distributed under the terms of the Creative Commons Attribution 4.0 International License (http://creativecommons.org/licenses/by/4.0/), which permits unrestricted use, distribution, and reproduction in any medium, provided you give appropriate credit to the original author(s) and the source, provide a link to the Creative Commons license, and indicate if changes were made. The Creative Commons Public Domain Dedication waiver (http://creativecommons.org/publicdomain/zero/1.0/) applies to the data made available in this article, unless otherwise stated. 
Regarding people seeking substance use treatment, depression is a particular concern because of its high prevalence and clinical implications [10-12]. Specifically, depression has been related to greater physical, psychological, and social impairments, poorer treatment adherence, and worse treatment outcomes [13, 14]. Importantly, several studies have found that depression decreases the likelihood of abstinence in people undergoing substance use treatment [15-17].

Previous literature has suggested the existence of common features between substance use disorders and depression. For example, studies have highlighted the key role of positive reinforcement in both conditions $[18,19]$. Positive reinforcement can be defined as the process by which a response is followed by a stimulus, and response probability increases. Positive reinforcers are fundamental in this process, and they can be defined as incentives, stimulus, and/or activities that are preferred for an individual. From a behavioural perspective, depression occurs when positive reinforcement for healthy behaviours decreases, there is a low availability of positive reinforcers in the environment, and/or when there is a lack of behavioural skills to achieve them [20]. In the case of SUDs, studies have found that people with SUDs are less engaged in non-drug-related activities and have less alternative positive reinforcers in their environment (e.g., social activities) [18, 21-23]. Indeed, previous research have demonstrated that engaging in alternative activities, as exercise or creative activities, was associated with reductions in substance use consumption [24, 25]. In this line, following the approach of behavioural economic interventions, Murphy et al. [26] found that adding a component addressing substance-free activities (academic, career-related, and leisure activities) to an alcohol brief motivational interviewing session for heavy drinking among college students, was associated with reductions in alcohol problems. Similarly, Reynolds et al. [27] integrated a BA approach within a standard college orientation program and found a significant reduction in the consequences associated with alcohol drinking (e.g., alcohol-related injuries, social and psychological problems).

Due to the high comorbidity between substance use and depression, and the impact of depression on substance use treatment outcomes, it is important to address both disorders simultaneously. There exist different treatments for depression and SUDs, as cognitive behavioural therapy (CBT) or contingence management interventions (CM) $[28,29]$. Although some preliminary findings indicate that these interventions have certain efficacy in treating both conditions, there is a need to continue developing and testing interventions for both disorders [30, 31].

Behavioural Activation (BA), which was originally conceptualized as a treatment for depression, is emerging as an option for SUDs. This intervention has its roots in the traditional behaviourism approach, but a renewed interest appeared since the study conducted by Jacobson et al. [32]. In such study, the authors isolated the BA component of cognitive therapy (CT) to determine whether BA by itself could be as effective as $\mathrm{CT}$ for depression treatment. Their results confirmed the equal effectiveness of $\mathrm{BA}$ and $\mathrm{CT}$ in reducing depressive symptoms, pointing out that $\mathrm{BA}$ is more parsimonious and less complex than CT. Nowadays, BA is considered a well-established and cost-effective intervention for depression [33, 34].

BA characteristics, and the focus on providing rewarding experiences in daily life different from substance use, make of this approach a potential intervention to increase substance use abstinence outcomes and to relapse prevention [35]. Since BA effectiveness has been widely demonstrated in depression treatment, we sought to extend previous findings by analyzing whether BA would improve comorbid substance use outcomes as well. Therefore, the aim of this review was to analyze the results of BA intervention on (i) substance use, abstinence, or relapse; and on (ii) depression symptom outcomes in individuals with substance use and depression.

\section{Method}

\section{Search strategy}

This systematic review followed the Preferred Reporting Items for Systematic Reviews and Meta-Analysis (PRISMA) statement [36], and the review protocol was registered with PROSPERO (CRD42016039412). The PRISMA checklist is provided in Additional file 1. The following electronic databases were used for the literature search, with alterations to the search strategy for specific databases: MEDLINE, PsycINFO, and Excerpta Medica DataBase (EMBASE). The literature search strategy for the three electronic databases, including any search limits used, is provided in Additional file 2. A search of reference lists of included studies and Google Scholar (first 200 citations published online between January 2000 and May 2018) was undertaken. We included studies published in English and Spanish, and all years available in the selected databases (up to May 2018).

\section{Study selection criteria Study characteristics}

The following study designs were included: (i) experimental studies (randomized controlled trials, quasi-randomized trials, controlled clinical trials); (ii) quasi-experimental studies (interrupted time series, before-and-after studies) and; (iii) observational studies (cohort studies and case-control studies). We excluded case series studies, research protocols, review articles, and non-interventional studies. 


\section{Participants}

Participants of included studies were: (i) adult substance users (age $\geq 18$ years); (ii) with depression. For the purpose of this review, substance users were defined as individuals who used substances assessed by a screening questionnaire (e.g., Substance Use Weekly Inventory) or as individuals who met criteria for SUD by a diagnostic interview (e.g., Structured Clinical Interview for Diagnostic and Statistical Manual of Mental Disorders; SCID-DSM). Substances included alcohol, tobacco, caffeine, cannabis, cocaine, heroin, amphetamines, ecstasy, synthetic drugs, and non-prescription use of legal drugs (e.g., morphine, codeine, benzodiazepines). People with depression were defined as individuals who experienced a depressive disorder assessed by a structured clinical interview conducted to internationally recognized standards (e.g., DSM) or depressive symptoms established by a validated screening measure (e.g., Beck Depression Inventory).

\section{Type of intervention}

Included studies were those examining the effect of face-to-face BA intervention on substance use and depression outcomes. To define BA features, Kanter et al. [37] reviewed the specific treatment components of BA and identified the following: activity monitoring, assessment of life goals and values, activity scheduling, skills training, relaxation training, contingency management, procedures targeting verbal behaviour, and procedures targeting avoidance. Despite the broad range of techniques used in BA interventions, they found that activity monitoring and scheduling were constant components across interventions.

Although there exist several conceptualizations of BA $[38,39]$, they all focus on behaviour change through the increase of positive reinforcement using strategies to encourage individuals to engage in adaptive and rewarding activities [40]. Therefore, in this review, we used the term 'BA' to cover all BA conceptualizations, including those studies where at least activity self-monitoring and scheduling were core elements of the intervention [37].

\section{Exclusion criteria}

Excluded studies were those in which: (i) participants had cognitive impairment; (ii) intervention was computerized or Internet-delivered; (iii) intervention did not include self-monitoring or activity scheduling; (iv) BA was only one component of CBT and not the core treatment element; and (v) both substance use and depression outcomes were not included.

\section{Outcomes}

Primary outcomes were: (i) substance use (at least one outcome related to substance use, abstinence, or relapse); and (ii) depressive symptoms.
Secondary outcomes were: (i) treatment adherence and retention; and (ii) number of quit attempts, use of other substances, motivation to quit, health-related conditions (e.g., diabetes, Human Immunodeficiency Virus, HIV), other mental health symptoms, and healthcare use. These variables were included as secondary outcomes since they have demonstrated to be predictors of treatment outcomes [41].

\section{Study selection}

Titles and abstracts retrieved by electronic searches were exported to reference management software (RefWorks) to remove duplicates. References were then exported to the online software tool Covidence for screening. Titles and abstracts were screened independently by two authors (CMV and UM). Disagreements were discussed by the two reviewers. The two reviewers (CMV and UM) performed independently full-text screening, data extraction, and quality assessment. Reasons for full text exclusion were recorded and documented in a PRISMA flow diagram (Fig. 1).

\section{Data extraction and analysis}

Data were extracted independently by CMV and UM using a data extraction form constructed in Microsoft Excel $2010^{\circ}$ : study identification features, study design, participant characteristics, sample size, intervention delivery mode, who delivered the intervention, whether the intervention was individual or group sessions, group size for group-based intervention, duration of intervention, number of sessions, length of sessions, treatment setting, depression outcomes, substance use outcomes, and, if reported, information on the components of the BA intervention. Discrepancies were resolved by discussion between the two reviewers.

\section{Assessment of risk of bias}

The quality of the studies that met eligibility criteria was independently assessed by two reviewers (CMV and $\mathrm{UM}$ ). Ratings were then reviewed to discuss discrepancies. Quality assessment was conducted using the Effective Public Health Practice Project Quality Assessment Tool (EPHPP). This is a generic tool used to evaluate a variety of intervention study designs such as randomized controlled trials (RCTs) and before-and-after studies. This tool has been considered suitable to be used in systematic reviews of effectiveness [42]. The tool assesses six domains: (i) selection bias, (ii) study design, (iii) confounders, (iv) blinding, (v) data collection method, and (vi) withdrawals/dropouts. The tool guidelines indicate that each domain can be rated as strong, moderate, or weak. Based on the total score studies can be assigned a quality rating of strong, moderate, or weak. 


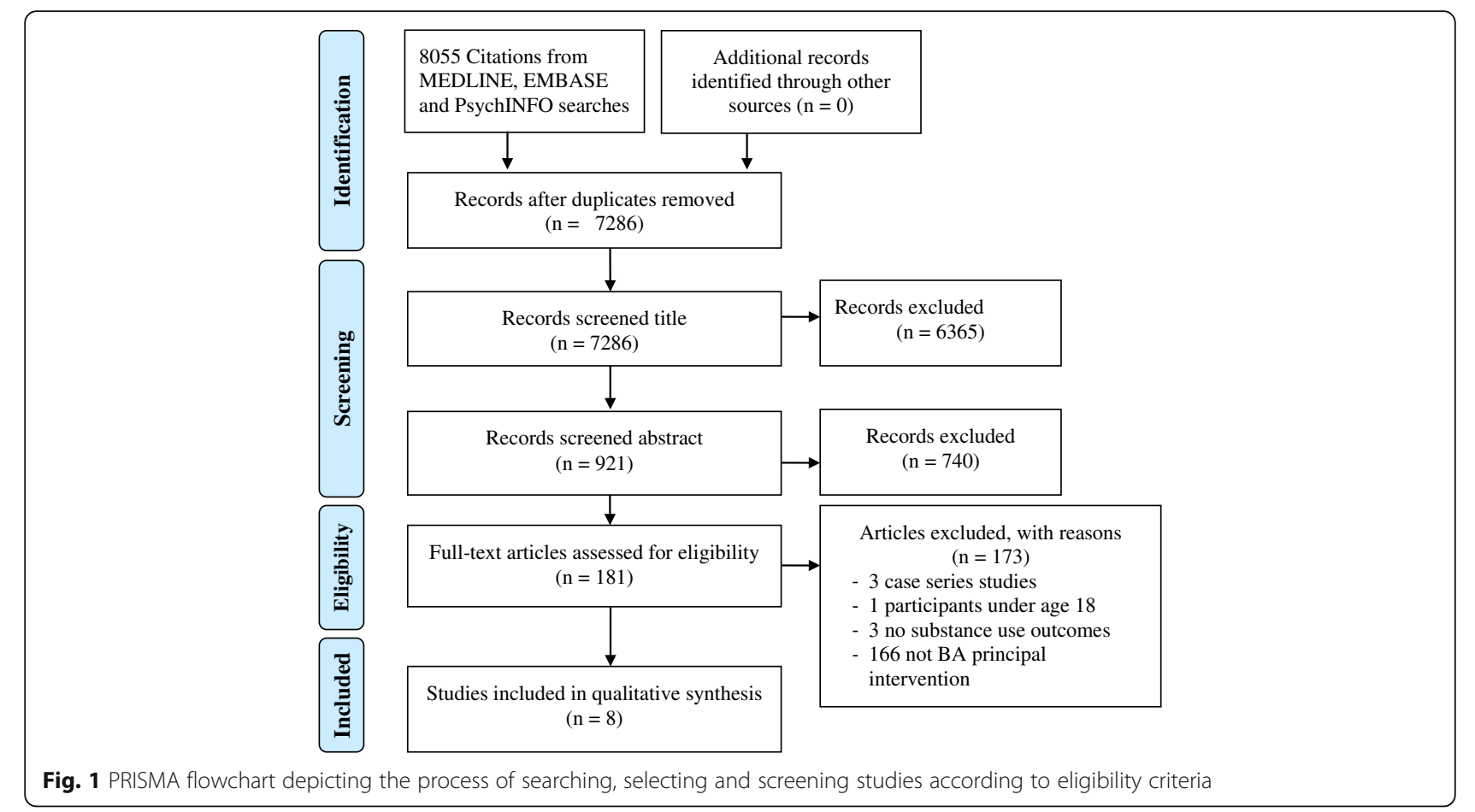

\section{Results}

A total of 7286 studies were identified after duplicates were removed. Once titles and abstracts were screened, 181 studies were selected for full text screening (Fig. 1). Finally, a total of eight studies met inclusion criteria and were included in the review [43-50].

\section{Study characteristics}

A complete description of study characteristics is provided in Table 1 . Of the eight included studies, six were conducted in the United States [43-45, 47-49], one in the United Kingdom [46], and one in Spain [50]. Six were RCTs [43, 45-47], and two were before-and-after studies [44, 48]. Regarding the type of substance assessed, three targeted smoking behaviour [43, 47, 50]; two targeted opiate dependence [44, 45]; two focused on alcohol or drug dependence [46, 49]; and finally, one on crystal methamphetamine abuse [48]. Six studies provided biochemical verification of substance use [43-45, $47,49,50]$. The assessment points ranged from baseline to 12 months post-intervention.

\section{Methodological quality assessment}

Overall, one study received a methodological quality rating of strong [49], six studies of moderate [43, 45-48, 50], and one study of weak [44]. The quality assessment ratings for each specific criterion and the assigned global rating are reported in Table 2. Study design and data collection dimensions were the main strengths of included studies, while blinding was the main weakness. Only in two studies $[47,49]$ participants and research staff assessing outcomes were blind to the study conditions. However, it is of note that blinding participants in behavioural intervention studies is often not feasible, as a result of the nature of the intervention.

The EPHPP tool provides two additional methodological dimensions (intervention integrity and analyses), which were also considered. Only three studies provided information about the intervention integrity by assessing the percentage of participants who received the intervention as intended: two were scored in the $80-100 \%$ category $[43,50]$; and one was scored in the less than $60 \%$ category [46]. With regard to the analysis component, all studies used intent-to-treat analyses as appropriate, except for Mimiaga et al. [48] who did not provide this information.

\section{Effects of BA intervention in substance use and depression outcomes}

Intervention descriptions and a summary of the main findings of the effects of BA on substance use and depression outcomes are reported in Table 3.

\section{Substance use outcomes}

Two of the six RCT included found significantly higher abstinence rates for BA compared to the control condition in each point assessment [47, 49]. Specifically, Daughters et al. [49] reported abstinence ORs of 2.2, 2.6, and 2.9 at 3, 6, and 12 months respectively in the BA condition. MacPherson et al. [47] also found significant 


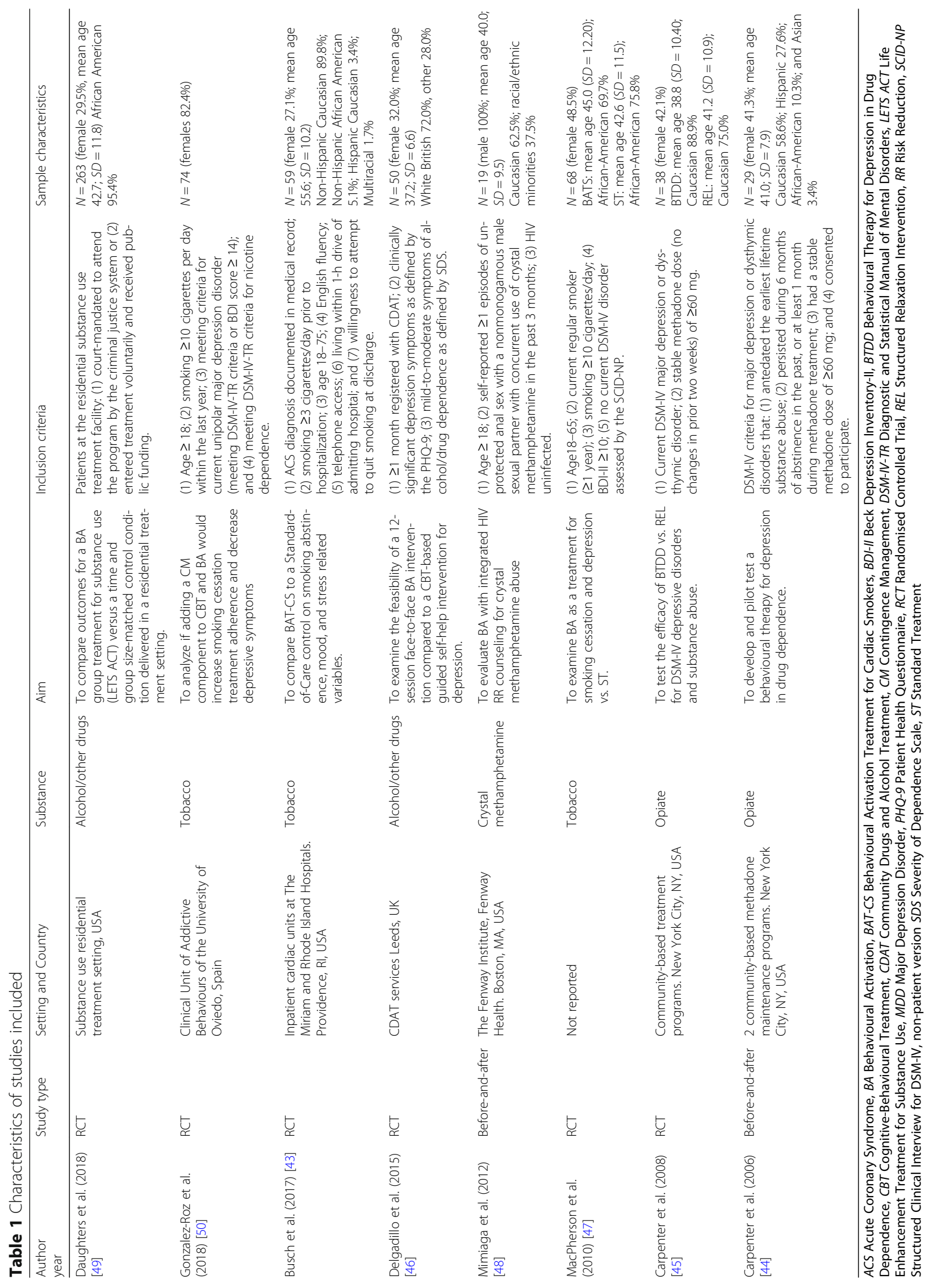


Table 2 Ratings of methodological quality by EPHPP tool

\begin{tabular}{llllllll}
\hline & Selection bias & Study design & Confounders & Blinding & Data collection & Withdrawals & Global rating \\
\hline Daughters et al. (2018) [49] & Strong & Strong & Strong & Strong & Strong & Strong & Strong \\
Gonzalez-Roz et al. (2018) [50] & Strong & Strong & Strong & Weak & Strong & Strong & Moderate \\
Busch et al. (2017) [43] & Strong & Strong & Strong & Weak & Strong & Strong & Moderate \\
Delgadillo et al. (2015) [46] & Moderate & Strong & Strong & Moderate & Strong & Weak & Moderate \\
Mimiaga et al. (2012) [48] & Strong & Moderate & Weak & Moderate & Strong & Strong & Moderate \\
MacPherson et al. (2010) [47] & Moderate & Strong & Strong & Strong & Strong & Weak & Moderate \\
Carpenter et al. (2008) [45] & Moderate & Strong & Moderate & Moderate & Strong & Moderate & Moderate \\
Carpenter et al. (2006) [44] & Weak & Moderate & Weak & Weak & Strong & Moderate & Weak \\
\hline
\end{tabular}

$\mathrm{OR}$ in the BA condition ( $\mathrm{OR}=4.0$ at 1 week post-quit; 2.06 at 4 weeks; 2.71 at 16 weeks, and 3.59 at 26 weeks).

No significant differences in abstinence rates were found between $\mathrm{BA}$ and the control conditions in the rest of the RCT included [43, 45, 46, 50]. However, in one study mean number of days to first lapse after discharge was significantly higher for BA when comparing to the control condition (62.4 vs. 31.8 days, respectively, $p$ $=.03$ ) [43]. Lastly, the study conducted by Delgadillo et al. [46], found $17 \%$ increase of days abstinent after treatment in the BA group. This indicates that there was a reduction in substance use in the BA group, whereas no change was detected in the control group [46]. Although there was a positive trend associated with the BA condition, differences were not statistically significant (Mean differences between-group effect size of $d=1.52, p$ $=.08$ ).

Regarding the two studies that compared pre- and postsubstance use rates the results were mixed. While Carpenter et al. [44] did not find changes in opiate and cocaine use after treatment, Mimiaga et al. [48] found a significant decrease from baseline to acute post-intervention and to 3 months post-intervention in the number of days of use in the past 30 days $(p=.010)$, in number of crystal methamphetamine episodes in the past 3 months $(p<.001)$, and in number of days experiencing drug-related problems during the past 30 days $(p=.005)$.

\section{Depression outcomes}

The majority of studies included in the present review found a significant improvement in depression symptoms over time [44-48, 50]. However, most studies showed equivalent results across treatment conditions $[45,46,49,50]$. Only one RCT [47] found a significant reduction in depression symptoms for those participants randomized to BA compared to the control group ( $B=$ $-1.99, S E=0.86, p=.02$ ). Moreover, depressive symptoms declined significantly from baseline to the 26-week post assigned quit-date $(B=-1.53, S E=0.68, p=.03)$.

Interestingly, one RCT study found a reduction in depression over time but only among abstainers regardless of the treatment condition [49]. Participants who remained abstinent at 12-month follow-up reported significantly fewer depressive symptoms, compared to substance users $(B=-5.74, \mathrm{SE}=1.65,95 \% \mathrm{CI}=-9.10$, 2.58). In addition, they found a significant decrease in depressive symptoms from pre-treatment to 12 -months post-treatment only in abstainers $(B=-0.43, \mathrm{SE}=0.11$, $95 \% \mathrm{CI}=-0.65,-0.22$ ).

\section{Discussion}

The aim of this systematic review was to examine if BA has an effect in reducing substance use and depression. Previous research has shown that reinforcement processes play a central role in the onset, maintenance, and recovery from depression [38] and SUDs [18]. Both disorders share features such as a reduced engagement in enjoyable non-drug-related activities/reinforcement or the presence of anhedonia, defined as a diminished interest/pleasure in response to previously rewarding activities [23, 51]. Since the main focus of BA is to increase healthy and rewarding activities [37], the potential use of BA in substance use treatment is justified.

Overall, the results of the present review were mixed. Although some studies indicated that BA reduced significantly substance use $[47,49]$ and depression [47], the effect sizes were moderate $[43,46]$. In addition, most of studies included did not reach statistical significance $[43,46,50]$. Since the majority of the studies were pilot $[43-45,47]$ or feasibility studies [46], they may be underpowered to detect significant differences. In fact, the only well powered RCT [49] showed that BA significantly reduced substance use.

Interestingly, BA has demonstrated its effectiveness for depression treatment [34]; although for people with substance use and depression, BA effect seems to be larger for substance use than for depression when compared against a control condition. It is possible that substance use outcomes (e.g., abstinence status) after treatment or during the follow-up period had an impact in depression outcomes, since previous research has found an association between substance use abstinence and depressive symptoms reduction [52-54]. Given that the majority of 


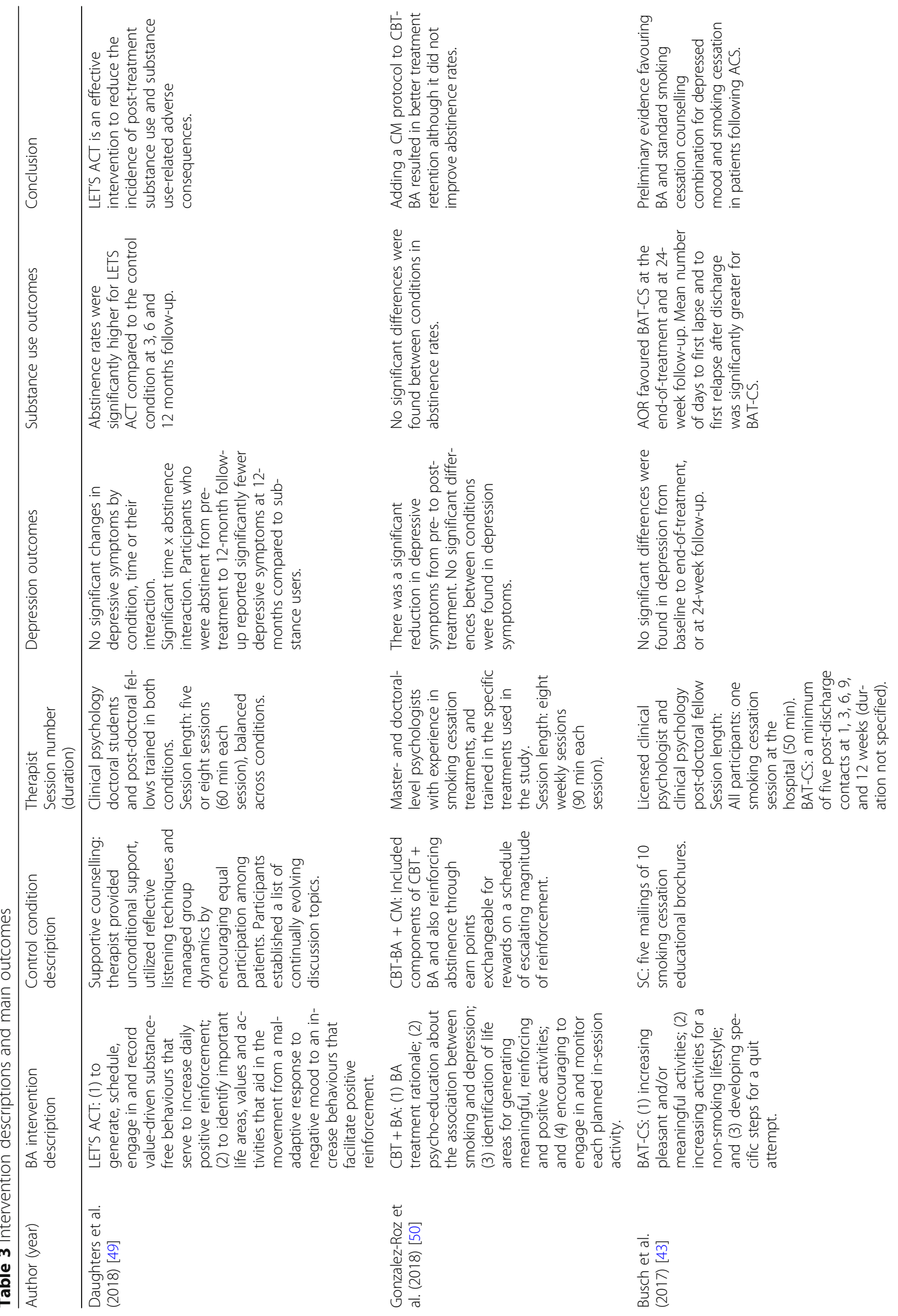




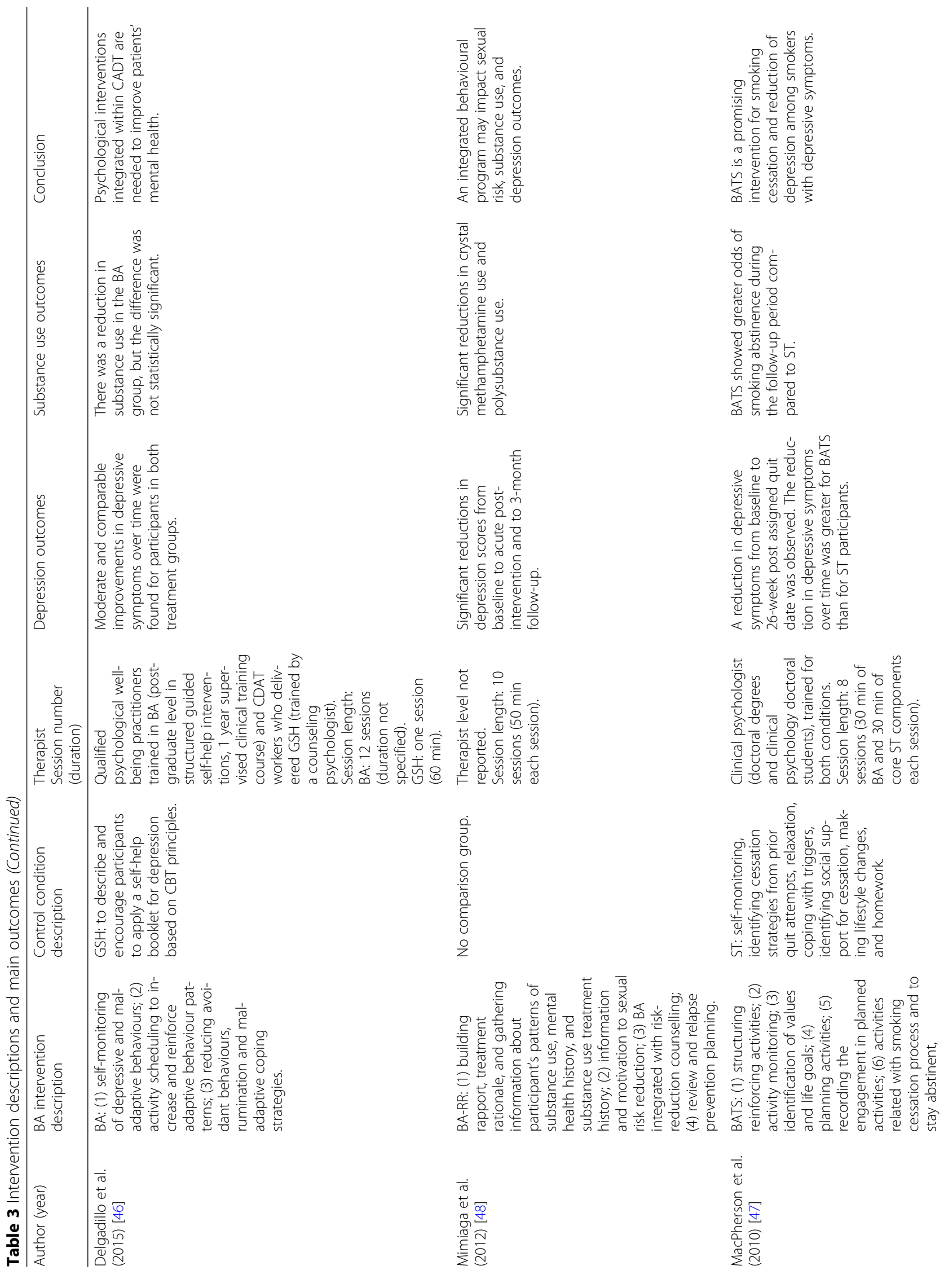




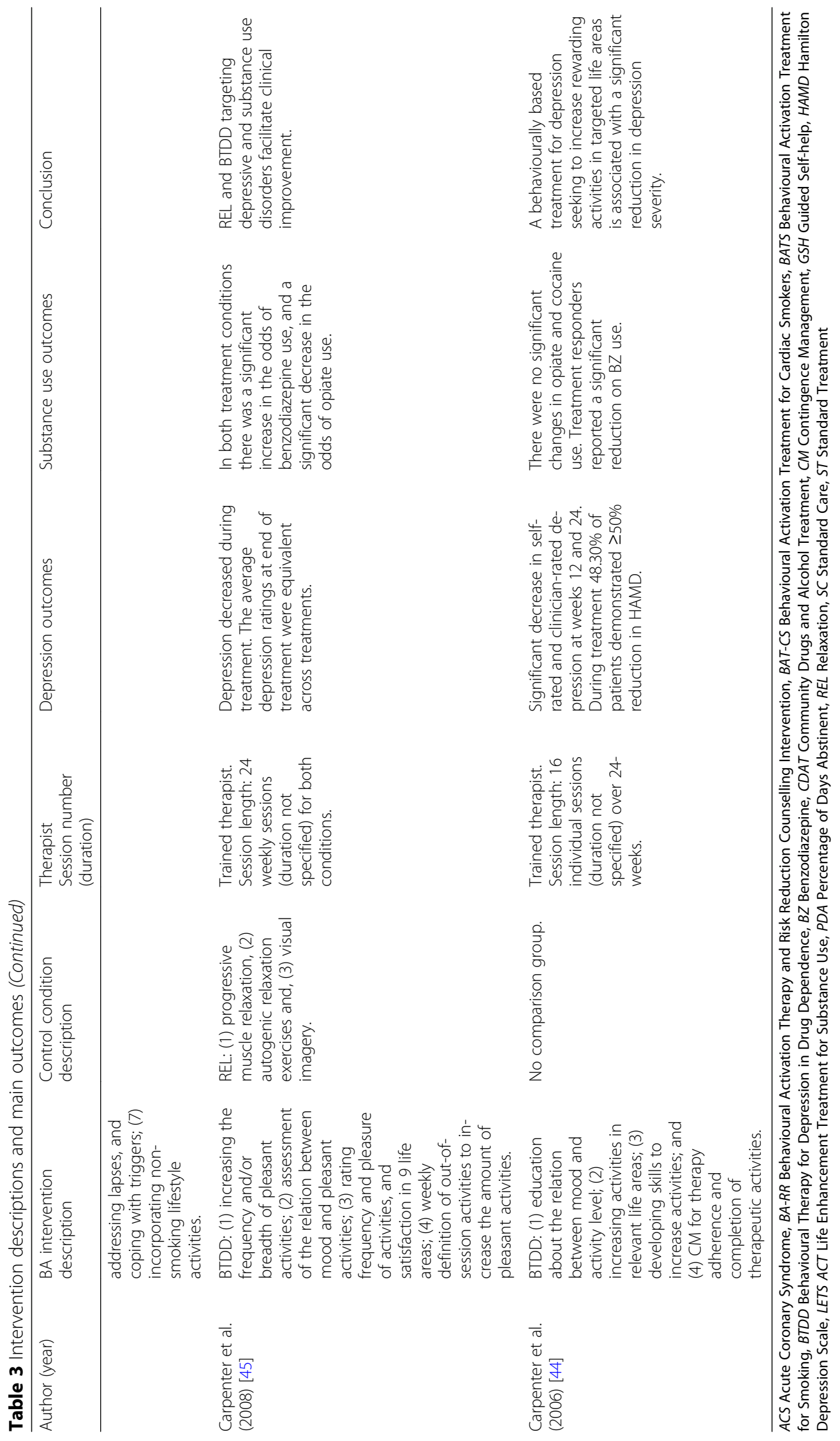


studies have analyzed substance use and depression outcomes separately, it would be interesting to analyze their interaction in future studies. Moreover, as suggested by Daughters et al. [49], research is needed to examine the effect of BA-based interventions on primary versus secondary depression in people with comorbid substance use and depression.

In addition, diverse factors could have influenced in the results found. One of them could be the heterogeneity of inclusion criteria for the different studies. For example, Busch et al. [43] and Mimiaga et al. [48] included participants with a wide range of baseline depression scores, from asymptomatic to individuals with severe symptomatology; whereas Carpenter et al. [44] and Carpenter et al. [45] only included participants with a DSM-IV diagnosis of major depression or dysthymic disorder. Measures for depression were also heterogeneous (e.g., Beck Depression Inventory-II; Hamilton Depression Scale; MontgomeryÅsberg Depression Rating Scale; Patient Health Questionnaire), as well as the target population (e.g., Acute Coronary Syndrome [ACS] patients, Human Immunodeficiency Virus [HIV] uninfected men who have sex with men), the treatment setting (e.g., inpatient cardiac units, community-based methadone maintenance programs; community drugs and alcohol treatment services), or the different stages of drug use treatment. Control conditions, type, length, and intensity also vary significantly across studies. Carpenter et al. [45] used a structured psychological treatment as a comparison group (e.g., 24 face-to-face weekly sessions of relaxation intervention), whereas Busch et al. [43] used a Standard-of-Care condition (e.g., one face-to-face session and five emails of printed educational material about smoking cessation). Baseline significant differences between groups could also have influenced the results. In fact, in the study conducted by Carpenter et al. [45], the Behavioural Therapy for Depression in Drug Dependence (BTDD) condition had a greater proportion of opiate users $(p \leq .03)$, and in the study conducted by Delgadillo et al. [46] baseline Severity of Dependence Scale was also significantly higher in the BA group $(p=.03)$. Finally, the studies included in this review used different $B A$ treatment approaches. Concretely, we found that two studies $[44,45]$ used an approach based on Lewinsohn et al. conceptualization [55], two studies $[46,48]$ used the Martell et al., BA approach [56], whereas the remaining four studies $[43,47,49,50]$ used a modified version of the brief behavioural activation treatment for depression (BATD) [39]. All these approaches are based on the principles of the behavioural model, share the use of behavioural strategies, and focus on behaviour change [40], but they have some differences. For example, the BA conceptualization of Lewinsohn et al. [55] focuses on assessing the relationship between mood and pleasant activity level, and on increasing the frequency of pleasant activities to facilitate positive interactions between the individual and the environment. The BA protocol of Martell et al. [38] focuses on behaviour functional analysis (examining antecedents and consequences) in order to identify behavioural avoidance patterns (e.g., avoid trying new activities or avoid attending social events/activities), and includes the use of strategies as mental rehearsal, periodic distraction or skill-training. In the case of the BATD model of Lejuez et al. [39], it focuses on increasing reinforcement for non-depressive behaviours (e.g., sport-related activities, social or leisure activities) emphasizing the personal value of these alternative behaviours. Further research is needed to determine if different BA treatment approaches have the same effects on depression and substance use outcomes.

Other limitations of this review include: first, the length of participants' follow-ups. Only one study included a 12 months follow-up [49], while the rest had the longest follow-up at 24 weeks post-intervention, which limited examining the long-term sustainability of treatment effects. Second, since BA effectiveness has been widely demonstrated in depression treatment [34, 57], we sought to investigate whether BA could improve not only depression, but also substance use outcomes. Thus, only studies that provided both outcomes were included. For this reason, we excluded two studies examining the effects of a BA intervention, named LETS Act!, on depression in standard inpatient substance abuse treatment $[58,59]$. Although they were excluded, the results of both studies suggest that the BA approach reduce depressive symptoms in this specific population, and one of them [59] also found a significantly lower percentage of individuals that dropped out of residential substance abuse treatment in the LETS Act! condition. These findings provide additional support to the positive effects of the BA approach.

Regarding the quality of the studies, it is of note that only one of the studies reached the qualification of strong methodological quality [49]. Further high-quality studies are needed to improve confidence in these findings and to confirm the positive effect of BA both in substance use outcomes and depression. In line with our results, a recent overview about cognitive-behavioural therapies for substance use and depression disorders conducted by Vujanovic et al. [28] showed that, despite the growing evidence supporting the effectiveness of integrated CBT for the treatment of co-occurring SUD-depression, the scarce of well-controlled studies limit their conclusions. Finally, six of the eight studies were conducted in the United States, which should be considered in the interpretation and generalization of results, as it has not been tested in others geographical and cultural settings.

Despite the limitations, this systematic review clearly described and followed internationally accepted standards 
for the process of identifying studies. In addition, despite that BA has demonstrated its effectiveness in the treatment of depression treatment, this review addresses its comorbidity with substance use. This is a novel and relevant topic since depression influences SUDs recovery and relapse. In addition, SUDs imply in many cases a lack of natural and alternative reinforcers and activities that are meaningful in life and provide a sense of purpose [35], which can have an impact in substance use-related behaviour change.

Although more research is needed to support the effectiveness of BA for the treatment of substance use and depression, the studies reviewed showed promising and suggestive data. Future studies are required to investigate the mechanisms of action of $\mathrm{BA}$, as well as possible moderator variables that can have impact in SUDs and depression outcomes. More research is also needed to investigate whether the BA model can just be applied to SUDs and whether the reward-related processes in SUDs and depression are as comparable as implied. For example, it is necessary to elucidate whether there are differences between anticipatory anhedonia (e.g., diminished subjective desire, interest, and anticipation of pleasant stimulus/ activity) and consummatory anhedonia (e.g., inability to experience pleasure in response to a pleasurable stimulus/activity) in SUDs and depression, and if this could influence treatment outcomes. Finally, future studies should be conducted on cost-effectiveness of this intervention approach, and on how BA can be implemented into clinical and community settings.

\section{Conclusions}

The results of this systematic review suggest that BA may help to improve substance use and depressive symptoms. However, research into BA in substance use and depression is at an early stage, and the majority of results are based on pilot studies with methodological limitations. Thus, they should be interpreted with caution. Given the high comorbidity of substance use and depressive symptoms, and the preliminary results indicating that BA may be a useful intervention for this population, more research is required to establish BA effectiveness. Future studies should be conducted adhering to standard reporting guidelines and using rigorous methodology including sample size calculations, adequate methods of randomization, intention-to-treat analysis, and longer follow-up periods.

In summary, BA is a promising option that could be easily integrated in substance use treatments due to its brevity and parsimony. BA could be implemented in treatment and community programs that make accessible and provide the opportunity to participate and engage in social, healthy, and cultural activities, offering more options for substance-free sources of reinforcement. Compromise and economic resources of governments and policymakers' result essential to make possible to deal with substance use and depression, as these problems have an enormous cost at personal, social, and economic levels.

\section{Additional files}

Additional file 1: PRISMA Checklist. (DOCX 29 kb)

Additional file 2: Literature search strategy. (PDF $460 \mathrm{~kb}$ )

\section{Abbreviations}

ACS: Acute coronary syndrome; BA: Behavioural activation; BATCS: Behavioural activation treatment for cardiac smokers; BDI-II: Beck depression inventory-II; BTDD: Behavioural therapy for depression in drug dependence; CBT: Cognitive behavioural treatment; CDAT: Community drugs and alcohol treatment; EPHPP: Effective public health practice project quality assessment tool; MDD: Major depression disorder; PHQ-9: Patient health questionnaire; RCT: Randomized controlled trial; REL: Structured relaxation intervention; RR: Risk reduction; SCID-NP: Structured clinical interview for dsm-iv, non-patient version; SDS: Severity of dependence scale; ST: Standard treatment; SUDs: Substance use disorders

\section{Acknowledgements}

This research was supported by the Spanish Ministry of Economy and Competiveness (Project reference: PSI2015-66755-R) and co-financed by FEDER (European Regional Development Fund; pluri-annual plan 2014-2020).

\section{Funding}

This research was supported by the Spanish Ministry of Economy and Competiveness (Project reference: PSI2015-66755-R) and co-financed by FEDER (European Regional Development Fund; pluri-annual plan 2014-2020). The authors alone are responsible for the content and writing of the article. Spanish Ministry of Economy and Competiveness had no role in the study design, collection, analysis or interpretation of the data, writing the manuscript, or the decision to submit the paper for publication.

Availability of data and materials

All data generated or analyzed during this study are included in this article.

\section{Authors' contributions}

All authors were involved in the drafting of the systematic review protocol. CMV and UM conducted the search, screening, study selection, and data extraction. ALD, EFR, and EB assisted with the interpretation of results. CMV drafted the initial manuscript and all authors contributed to subsequent revisions of the manuscript. All authors have contributed to and approved the final version of the manuscript.

\section{Ethics approval and consent to participate}

This is a review of published studies. No ethical approval was necessary, but all included studies were conducted with ethical approval and consent.

\section{Consent for publication}

Not applicable.

\section{Competing interests}

The authors declare that they have no competing interests.

\section{Publisher's Note}

Springer Nature remains neutral with regard to jurisdictional claims in published maps and institutional affiliations. 


\section{Author details}

${ }^{1}$ Smoking Cessation and Addictive Disorders Unit, Department of Clinical Psychology and Psychobiology, Faculty of Psychology, University of Santiago de Compostela, Santiago de Compostela, Spain. ${ }^{2}$ Tobacco Research and Intervention Program. Department of Health Outcomes and Behaviour, $\mathrm{H}$. Lee Moffitt Cancer Center, Fl, Tampa, USA. ${ }^{3}$ Department of Psychology and Sociology, University of Zaragoza, Zaragoza, Spain.

Received: 12 March 2018 Accepted: 21 September 2018

Published online: 29 September 2018

\section{References}

1. Whiteford HA, Ferrari AJ, Degenhardt L, Feigin V, Vos T. The global burden of mental, neurological and substance use disorders: an analysis from the global burden of disease study 2010. PLoS One. 2015;10:e0116820.

2. Han B, Olfson M, Mojtabai R. Depression care among adults with cooccurring major depressive episodes and substance use disorders in the United States. J Psychiatr Res. 2017;91:47-56.

3. Hasin DS, Kerridge BT, Saha TD, Huang B, Pickering R, Smith SM, et al. Prevalence and correlates of DSM-5 Cannabis use disorder, 2012-2013: findings from the National Epidemiologic Survey on alcohol and related conditions-III. Am J Psychiatry. 2016;173:588-99.

4. Kessler RC, Chiu WT, Demler O, Walters EE. Prevalence, severity, and comorbidity of 12-month DSM-IV disorders in the National Comorbidity Survey Replication. Arch Gen Psychiatry. 2005;62:617-27.

5. Prior K, Mills K, Ross J, Teesson M. Substance use disorders comorbid with mood and anxiety disorders in the Australian general population. Drug Alcohol Rev. 2017:36:317-24.

6. Rush B, Koegl CJ. Prevalence and profile of people with co-occurring mental and substance use disorders within a comprehensive mental health system. Can J Psychiatr. 2008;53:810-21.

7. Lai HMX, Cleary M, Sitharthan T, Hunt GE. Prevalence of comorbid substance use, anxiety and mood disorders in epidemiological surveys, 1990-2014: a systematic review and meta-analysis. Drug Alcohol Depend. 2015:154:1-13.

8. Grant BF, Saha TD, Ruan WJ, Goldstein RB, Chou SP, Jung J, et al. Epidemiology of DSM-5 drug use disorder. JAMA Psychiatry. 2016:73:39-47.

9. Cooper J, Borland R, Yong H-H, Fotuhi O. The impact of quitting smoking on depressive symptoms: findings from the international tobacco control four-country survey. Addiction. 2016;111:1448-56.

10. Goldner EM, Lusted A, Roerecke M, Rehm J, Fischer B. Prevalence of Axis-1 psychiatric (with focus on depression and anxiety) disorder and symptomatology among non-medical prescription opioid users in substance use treatment: systematic review and meta-analyses. Addict Behav. 2014;39:520-31

11. Landheim AS, Bakken K, Vaglum P. Impact of comorbid psychiatric disorders on the outcome of substance abusers: a six year prospective follow-up in two Norwegian counties. BMC Psychiatry. 2006:6:44.

12. Piper ME, Smith SS, Schlam TR, Fleming MF, Bittrich AA, Brown JL, et al. Psychiatric disorders in smokers seeking treatment for tobacco dependence: relations with tobacco dependence and cessation. J Consult Clin Psychol. 2010:78:13-23.

13. McKay JR, Pettinati HM, Morrison R, Feeley M, Mulvaney FD, Gallop R. Relation of depression diagnoses to 2-year outcomes in cocaine-dependent patients in a randomized continuing care study. Psychol Addict Behav. 2002;16:225-35.

14. Teesson M, Mills K, Ross J, Darke S, Williamson A, Havard A. The impact of treatment on 3 years' outcome for heroin dependence: findings from the Australian treatment outcome study (ATOS). Addiction. 2008:103:80-8.

15. Dodge R, Sindelar J, Sinha R. The role of depression symptoms in predicting drug abstinence in outpatient substance abuse treatment. J Subst Abus Treat. 2005;28:189-96.

16. Glasner-Edwards S, Marinelli-Casey P, Hillhouse M, Ang A, Mooney LJ, Rawson $\mathrm{R}$, et al. Depression among methamphetamine users: association with outcomes from the methamphetamine treatment project at 3-year follow-up. J Nerv Ment Dis. 2009:197:225-31.

17. Stepankova L, Kralikova E, Zvolska K, Pankova A, Ovesna P, Blaha M, et al. Depression and smoking cessation: evidence from a smoking cessation clinic with 1-year follow-up. Ann Behav Med. 2017;51:454-63.

18. Higgins ST, Heil SH, Lussier JP. Clinical implications of reinforcement as a determinant of substance use disorders. Annu Rev Psychol. 2004;55:431-61.
19. Dimidjian S, Barrera M, Martell C, Muñoz RF, Lewinsohn PM. The origins and current status of behavioral activation treatments for depression. Annu Rev Clin Psychol. 2011;7:1-38.

20. Jacobson NS, Martell CR, Dimidjian S. Behavioral activation treatment for depression: returning to contextual roots. Clin Psychol Sci Pract. 2006;8:255-70.

21. Audrain-McGovern J, Rodriguez D, Rodgers K, Cuevas J. Declining alternative reinforcers link depression to young adult smoking. Addiction. 2011;106:178-87.

22. Lubman DI, Yücel M, Kettle JWL, Scaffidi A, MacKenzie T, Simmons JG, et al. Responsiveness to drug cues and natural rewards in opiate addiction. Arch Gen Psychiatry. 2009;66:205.

23. Magidson JF, Robustelli BL, Seitz-Brown CJ, Whisman MA. Activity enjoyment, not frequency, is associated with alcohol-related problems and heavy episodic drinking. Psychol Addict Behav. 2017;31:73-8.

24. Rogers RE, Higgins ST, Silverman K, Thomas CS, Badger GJ, Bigelow G, et al. Abstinence-contingent reinforcement and engagement in nondrug-related activities among illicit drug abusers. Psychol Addict Behav. 2008;22:544-50

25. Correia CJ, Benson TA, Carey KB. Decreased substance use following increases in alternative behaviors: a preliminary investigation. Addict Behav. 2005:30:19-27.

26. Murphy JG, Dennhardt AA, Skidmore JR, Borsari B, Barnett NP, Colby SM, et al. A randomized controlled trial of a behavioral economic supplement to brief motivational interventions for college drinking. J Consult Clin Psychol. 2012;80:876-86.

27. Reynolds EK, Macpherson L, Baruch DE, Tull MT, Lejuez CW. Integration of the Brief Behavioral Activation Treatment for Depression (BATD) Into a College Orientation Program: Depression and Alcohol Outcomes. J Couns Psychol. 2011;58:555-64

28. Vujanovic AA, Meyer TD, Heads AM, Stotts AL, Villarreal YR, Schmitz JM. Cognitive-behavioral therapies for depression and substance use disorders: an overview of traditional, third-wave, and transdiagnostic approaches. Am J Drug Alcohol Abuse. 2017:43:402-15.

29. Murphy SM, McDonell MG, McPherson S, Srebnik D, Angelo F, Roll JM, et al. An economic evaluation of a contingency-management intervention for stimulant use among community mental health patients with serious mental illness. Drug Alcohol Depend. 2015;153:293-9.

30. Hides L, Samet S, Lubman DI. Cognitive behaviour therapy (CBT) for the treatment of co-occurring depression and substance use: current evidence and directions for future research. Drug Alcohol Rev. 2010;29:508-17.

31. Hesse M. Integrated psychological treatment for substance use and comorbid anxiety or depression vs. treatment for substance use alone. A systematic review of the published literature. BMC Psychiatry. 2009;9:6.

32. Jacobson NS, Dobson KS, Truax PA, Addis ME, Koerner K, Gollan JK, et al. A component analysis of cognitive-behavioral treatment for depression. J Consult Clin Psychol. 1996;64:295-304.

33. Richards DA, Ekers D, McMillan D, Taylor RS, Byford S, Warren FC, et al. Cost and outcome of Behavioural activation versus cognitive Behavioural therapy for depression (COBRA): a randomised, controlled, non-inferiority trial. Lancet. 2016;388:871-80.

34. Ekers D, Webster L, Van Straten A, Cuijpers P, Richards D, Gilbody S. Behavioural activation for depression; an update of meta-analysis of effectiveness and sub group analysis. PLoS One. 2014;9:e100100.

35. McKay JR. Making the hard work of recovery more attractive for those with substance use disorders. Addiction. 2017;112:751-7.

36. Moher D, Liberati A, Tetzlaff J, Altman DG. PRISMA group. Preferred reporting items for systematic reviews and meta-analyses: the PRISMA statement PLoS Med. 2009;6:e1000097.

37. Kanter JW, Manos RC, Bowe WM, Baruch DE, Busch AM, Rusch LC. What is behavioral activation?A review of the empirical literature. Clin Psychol Rev. 2010;30:608-20.

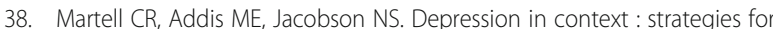
guided action. New York: W.W. Norton; 2001.

39. Lejuez CW, Hopko DR, Hopko SD. A brief behavioral activation treatment for depression. Treatment manual Behav Modif. 2001;25:255-86.

40. Hopko DR, Lejuez CW, Ruggiero KJ, Eifert GH. Contemporary behavioral activation treatments for depression: Procedures, principles, and progress. Clin Psychol Rev. 2003;23:699-717. https://doi.org/10.1016/S0272 7358(03)00070-9.

41. DiClemente CC. Motivation for change: implications for substance abuse treatment. Psychol Sci. 1999;10:209-13. 
42. Armijo-Olivo S, Stiles CR, Hagen NA, Biondo PD, Cummings GG. Assessment of study quality for systematic reviews: a comparison of the Cochrane collaboration risk of Bias tool and the effective public health practice project quality assessment tool: methodological research. J Eval Clin Pract. 2012;18:12-8.

43. Busch AM, Tooley EM, Dunsiger S, Chattillion EA, Srour JF, Pagoto SL, et al. Behavioral activation for smoking cessation and mood management following a cardiac event: results of a pilot randomized controlled trial. BMC Public Health. 2017;17:323.

44. Carpenter KM, Aharonovich E, Smith JL, Iguchi MY, Nunes EV. Behavior therapy for depression in drug dependence (BTDD): results of a stage la therapy development pilot. Am J Drug Alcohol Abuse. 2006;32:541-8.

45. Carpenter KM, Smith JL, Aharonovich E, Nunes EV. Developing therapies for depression in drug dependence: results of a stage 1 therapy study. Am J Drug Alcohol Abuse. 2008;34:642-52.

46. Delgadillo J, Gore S, Ali S, Ekers D, Gilbody S, Gilchrist G, et al. Feasibility randomized controlled trial of cognitive and behavioral interventions for depression symptoms in patients accessing drug and alcohol treatment. J Subst Abus Treat. 2015;55:6-14.

47. MacPherson L, Tull MT, Matusiewicz AK, Rodman S, Strong DR, Kahler CW, et al. Randomized controlled trial of behavioral activation smoking cessation treatment for smokers with elevated depressive symptoms. J Consult Clin Psychol. 2010;78:55-61.

48. Mimiaga MJ, Reisner SL, Pantalone DW, O'Cleirigh C, Mayer KH, Safren SA. A pilot trial of integrated behavioral activation and sexual risk reduction counseling for HIV-uninfected men who have sex with men abusing crystal methamphetamine. AIDS Patient Care STDs. 2012;26:681-93.

49. Daughters SB, Magidson JF, Anand D, Seitz-Brown CJ, Chen Y, Baker S. The effect of a behavioral activation treatment for substance use on posttreatment abstinence: a randomized controlled trial. Addiction. 2018;113: 535-44.

50. González-Roz A, Secades-Villa R, Alonso-Pérez F. Effects of combining contingency management with behavioral activation for smokers with depression. Addict Res Theory. 2018:1-8.

51. Hatzigiakoumis DS, Martinotti G, Di GM, Janiri L. Anhedonia and substance dependence: clinical correlates and treatment options. Front psychiatry. 2011;2:10.

52. Mathew AR, Robinson JD, Norton PJ, Cinciripini PM, Brown RA, Blalock JA. Affective trajectories before and after a quit attempt among smokers with current depressive disorders. Nicotine Tob Res. 2013;15:1807-15.

53. Driessen $\mathrm{M}$. The course of anxiety, depression and drinking behaviours after completed detoxification in alcoholics with and without comorbid anxiety and depressive disorders. Alcohol Alcohol. 2001;36:249-55.

54. ludicello JE, Woods SP, Vigil O, Cobb Scott J, Cherner M, Heaton RK, et al. Longer term improvement in neurocognitive functioning and affective distress among methamphetamine users who achieve stable abstinence. J Clin Exp Neuropsychol. 2010;32:704-18.

55. Lewinsohn PM, Sullivan JM, Grosscup SJ. Changing reinforcing events: an approach to the treatment of depression. Psychother Theory, Res Pract. 1980:17:322-34.

56. Martell CR, Dimidjian S, Herman-Dunn R. Behavioral activation for depression : a clinician's guide: Guilford Press; 2010

57. Mazzucchelli T, Kane R, Rees C. Behavioral activation treatments for depression in adults: a meta-analysis and review. Clin Psychol Sci. 2009;16: 383-411.

58. Daughters SB, Braun AR, Sargeant MN, Reynolds EK, Hopko DR, Blanco C, et al. Effectiveness of a brief behavioral treatment for inner-city illicit drug users with elevated depressive symptoms: the life enhancement treatment for substance use (LETS act!). J Clin Psychiatry. 2008;69:122-9.

59. Magidson JF, Gorka SM, MacPherson L, Hopko DR, Blanco C, Lejuez CW, et al. Examining the effect of the life enhancement treatment for substance use (LETS ACT) on residential substance abuse treatment retention. Addict Behav. 2011;36:615-23.

Ready to submit your research? Choose BMC and benefit from:

- fast, convenient online submission

- thorough peer review by experienced researchers in your field

- rapid publication on acceptance

- support for research data, including large and complex data types

- gold Open Access which fosters wider collaboration and increased citations

- maximum visibility for your research: over $100 \mathrm{M}$ website views per year

At BMC, research is always in progress.

Learn more biomedcentral.com/submissions 